\title{
Playground Social Interaction Analysis using Bespoke Wearable Sensors for Tracking and Motion Capture
}

\author{
Behzad M. Heravi* \\ Department of Computer Science, Faculty of Engineering \\ Sciences \\ University College London, UK \\ b.heravi@ucl.ac.uk \\ Stephen Hailes \\ Department of Computer Science, Faculty of Engineering \\ Sciences \\ University College London, UK \\ s.hailes@cs.ucl.ac.uk
}

\begin{abstract}
Unstructured play ${ }^{1}$ is considered important for the social, physical and cognitive development of children. Traditional observational research examining play behaviour at playtime (recess) has been hampered by challenges in obtaining reliable data and in processing sufficient quantities of that data to permit credible inferences to be drawn. The emergence of wearable wireless sensor technology makes it possible to study individual differences in childhood social behaviour based on collective movement patterns during playtime. In this work, we introduce a new method to enable simultaneous collection of GNSS/IMU data from a group of children interacting on a playground. We present a detailed description of system development and implementation before going on to explore methods of characterising social groups based on collective movement recording and analysis. A case study was carried out for a class of 7-8 year old children in their school playground during 10 episodes of unstructured play. A further 10 play episodes were monitored in the same space following the introduction of large, loose play materials. This study design allowed us to observe the effect of an environmental intervention on social movement patterns. Sociometric analysis was conducted for comparison and validation. This successful case study demonstrates that sensor based movement data can be used to explore children's social behaviour during naturalistic play.
\end{abstract}

* These authors are co-first authors of this work

${ }^{1}$ Permission to make digital or hard copies of all or part of this work for personal or classroom use is granted without fee provided that copies are not made or distributed for profit or commercial advantage and that copies bear this notice and the full citation on the first page. Copyrights for components of this work owned by others than the author(s) must be honored. Abstracting with credit is permitted. To copy otherwise, or republish, to post on servers or to redistribute to lists, requires prior specific permission and/or a fee. Request permissions from Permissions@ acm.org. MOCO '18, June 28-30, 2018, Genoa, Italy

(C) 2018 Copyright is held by the owner/author(s). Publication rights licensed to ACM. ACM ISBN 978-1-4503-6504-8/18/06 . \$15.00

https://doi.org/10.1145/3212721.3212818

\author{
Jenny L. Gibson* \\ Centre for research on Play in Education, Development \\ and Learning (PEDAL), Faculty of Education \\ University of Cambridge, UK \\ jlg53@cam.ac.uk \\ David Skuse \\ Behavioural Sciences Unit, Institute of Child Health \\ University College London, UK \\ d.skuse@ucl.ac.uk
}

\section{CCS CONCEPTS}

- Computer systems organization $\rightarrow$ Embedded systems; Sensors - Information systems $\rightarrow$ Information systems applications; Spatial-Temporal systems

\section{KEYWORDS}

Curve Similarity, Social Interactions, Play, Childhood Social Behaviour, Wearable Sensors, Tracking and Motion Capture

ACM Reference format:

Behzad M. Heravi, Jenny L. Gibson, Stephen Hailes, David Skuse. 2018. In Proceedings of ACM Movement and Computing conference, Genoa, Italy, June 2018 (MOCO'18), 8 pages. DOI: $10.1145 / 3212721.3212818$

\section{INTRODUCTION}

School playgrounds are significant spaces in children's lives but their importance as a context for social, physical and cognitive development is under-researched and poorly understood [8]. In particular, social group structure and dynamics are thought to be significantly related to childhood wellbeing but have rarely been studied in naturalistic play episodes. One reason for this is that observation of children's unstructured social interactions can be challenging to implement, requiring highly resource intensive methods that are difficult scale to the group level (e.g. see [1]). Developments in wearable wireless sensor technology raise the possibility of capturing important social behaviours 'in the wild' [11], allowing the study of group behaviour in natural contexts. There is an emerging literature concerning the use of such technologies to study individual differences in childhood social behaviour. Veiga and colleagues have used RFID to establish that frequent proximity to other children during play is a good predictor of social competence [9]. In the present study we were motivated include movement data into the study of play as another source of social insights. For example, Moreno and colleagues have demonstrated using simulation, how social roles during 
games can be inferred from movement data alone [7]. Inspired by these studies, as well as by innovative movement studies of nonhuman animal social behaviour [10], our guiding research question was, "how can we use wireless technology to capitalise on the rich potential of playground behaviour as a way to explore and understand childhood social development?"

In the current paper we introduce a new method to enable simultaneous collection of Global Navigation Satellite System (GNSS) and Inertial Measurement Unit (IMU) data from a group of children interacting on a playground, on repeated occasions. We present a detailed description of the system implementation before going on to use a descriptive case study approach to explore methods of characterising the structure of social groups based on the resulting data. Structures of interest include friendship 'cliques' and segregation by gender or other player attributes. We define 3 types of gameplay based on our observations and formulise movement similarity methods to classify social gameplays. Through cluster analysis we firstly validate our results against sociometric data and then compare pre- and post-intervention results in terms of gender gap and social groups.

The specific aims of the study were to 1) construct a user-friendly system of wearable sensors to track children's movements and interactions on the playground, and 2) develop ways to use the resulting sensor data to characterise social play behaviours. These aims are important for the field of psychology, given the potential to reduce costs, and increase replicability of research into social play outside of laboratory conditions.

\section{METHODS}

\subsection{The Case Study}

The study was approved by the institutional ethical review board. As participants were minors, informed consent was sought from those with parental responsibility. An information session was held at the school to answer any questions and concerns that children, parents and teachers had about the study. Most potential participants were comfortable with the technology and children asked interesting questions about privacy and encryption of data. Permission to participate was given in all but one case (18 out of 19 children joined the study).

The project team was interdisciplinary, with team members from Computer Science, Education, Psychology, Psychiatry and Playwork. The team devised a case study approach to the research, with an emphasis on the development of the wearable sensors, the feasibility of using them with children, and qualitatively evaluating whether resulting data would be likely to be useful for the study of play. There were 18 participants, 9 girls and 9 boys all from one year-3 class in an urban, Primary School in the UK (age 7-8 years).

The project was introduced to the children during a lesson, and they were invited to ask questions of the researchers. Children were involved in personalising the wearables using pens and fabric paints. The aim of these activities was to raise interest and engagement with the project.
The basic idea of the study was to collect location data while the children were engaged in free-play on the school playground. We introduced a basic 'loose parts play' intervention to provide information about how changes to the play environment influence children's interactions. Loose parts play introduces to the play space loose materials that have an ambiguous affordance [3]; the aim is to foster creativity and collaboration. In the baseline condition (10 sessions) children were left to play without any toys or other materials. In the intervention condition (10 sessions) loose materials, balls, and access to 2 large fixed play structures were provided and children were facilitated by two adult play workers.

Sensor data were collected over 27 sessions of at least 45 minutes each; of these, 7 initial sessions were assigned as pilot or habituation, 10 sessions as 'baseline' and 10 sessions as 'intervention'. Due to the constraints of the school day it was not possible to randomise the hour assigned for data collection, instead we collected data at the same time each day (12:00-13:00). The playground (Figure 1) is $24 \times 35$ metres and was divided into zones defining areas of interest (shown in green in Figure 1), inaccessible zones (shown in red and dark red) and boundary zones (shown in blue and orange). During data collection, recordings were made using two cameras that covered the area, for ground truth data collection and validation.

\subsection{Wearable Sensors and Devices}

In order to analyse collective movement patterns of children in the playground and to study their social interactions, sub-metre positioning accuracy and synchronised measurement for each child during the playtime were required. The technology also had to be unobtrusive and not inhibitory to children and their play. Commercially off-the-shelf (COTS) equipment was not able to meet these requirements at reasonable cost. COTS positioning devices in wearable form do not offer sub-meter accuracy. Such accuracy can only be achieved by recording raw satellite data and post processing using real-time kinematic corrections. Therefore, two bespoke sensors/devices were designed and developed inhouse for this experiment: (i) a wearable head-mounted sensor with IMU and GNSS for precise positioning; and (ii) a wearable shoe-mounted IMU sensor for activity monitoring, motion capture and GNSS augmentation (see Figures 2 and 3).

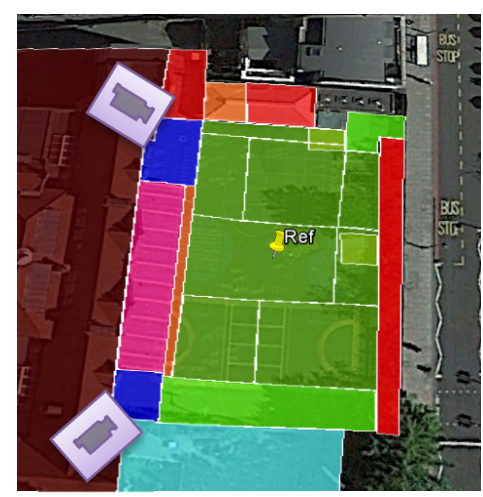

Figure 1: The Playground 


\subsubsection{GNSS/IMU Sensor}

The GNSS/IMU sensor incorporates a raw data GNSS module and active antenna supporting concurrent reception of multiple satellite systems (GPS, GLONASS, BeiDou and Galileo). Raw GNSS data include carrier phase, code pseudorange and Doppler measurements. Logging of raw GNSS data allows off-line processing and cancellation of carrier-phase ambiguity and ionospheric noise using base station data. However, depending on the location of the sensor, obstructions such as playground trees, walls, metal fencing, or nearby buildings interrupted the line-ofsight between the GNSS antenna and the satellites and, as a result, compromised the positioning accuracy. To counter this, concurrent information from multiple satellite systems, and the capture of both raw and real-time position-velocity-time (PVT) GPS data was used to increase redundancy improve the positioning precision. The sensor is also equipped with 9-axis IMU system combining a 3-axis accelerometer, 3-axis gyroscope, and 3-axis magnetometer. The sensor uses Bluetooth Low Energy (BLE) for wireless connectivity for configuration and data streaming and a micro-SD card is used to log the IMU and GNSS data. Highly accurate satellite time is used to synchronise the sensors. Figure 2 shows the in-house built GNSS/IMU Sensors and antenna.

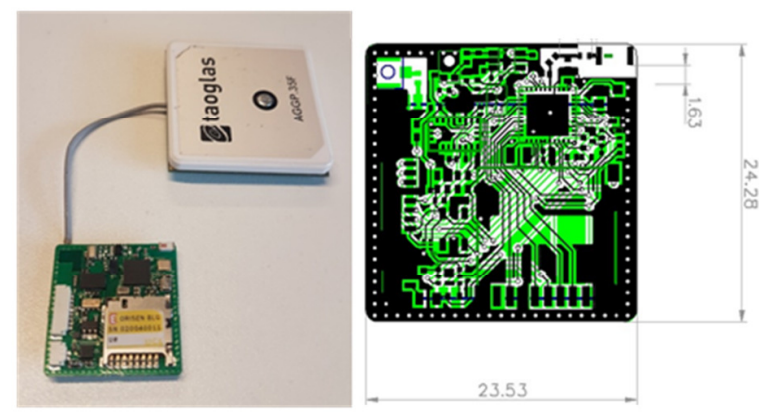

Figure 2: Bespoke GNSS/IMU Sensors

Wearable protective shells for the GNSS/IMU sensors were designed, 3D modelled and printed. The protective shell allows the sensor, antenna and battery to safely reside beneath the brim of a baseball cap. This provides a flat horizontal space for the GNSS antenna which provides the optimum line-of-sight (LoS) to satellites. Figure 3 shows the 3D printed models of the headmounted (baseball cap brim) protective shell for the GNSS/IMU sensors.

\subsubsection{Shoe Flap IMU Sensor}

Foot-worn sensors can provide step-detection, activity monitoring and movement analysis, and can be used to augment position calculations $[12,13]$. In this project, a wearable IMU sensor was designed and developed in-house firstly to improve positioning accuracy and secondly to measure activity levels during playtime. During periods in which there is no clear line-of-sight view of satellites (e.g. when close to the school building or under playground trees), step detection using foot-worn IMU sensors can maintain the positioning accuracy at sub-metre precision levels. The bespoke sensors include 9-axis IMU sensors for 3 degree-of-freedom (DoF) motion capture and BLE wireless connectivity for configurations and synchronisation with real time. Figure 4 shows the miniature IMU sensor. Again, the IMU sensor and its battery is placed into a protective shell in shape of a shoe flap that can be worn on top of shoes with or without shoe laces. Figure 5 shows the designed and 3D printed shoe-flap for the IMU sensor.

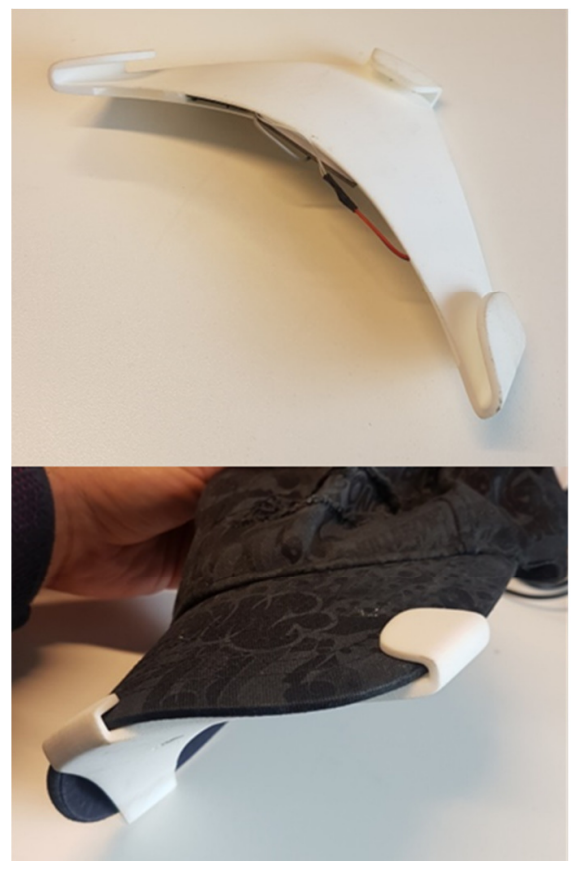

Figure 3: 3D printed head-mounted protective shell for the GNSS/IMU Sensors
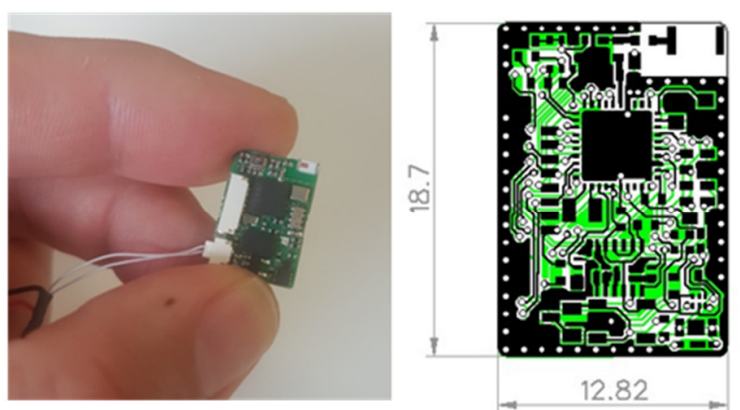

Figure 4: Bespoke IMU Sensors

\subsection{Sociometric Analysis}

The social structure of the class was analysed using a sociometric nomination procedure commonly used in psychology studies [2]. The idea is that reports of relationships (in this case friendship) are sought from each individual and represented as a weighted 
adjacency matrix. This can be analysed to give insight into the social organisation of the group. For example, the most and least popular group members can be identified.

Each child was interviewed on a one-to-one basis in a quiet area of the school and was asked to nominate their 3 most preferred classmates in order of preference. Photographs of all participating classmates were used to support nominations, and children were assured that nominations were confidential. Self-nominations were not permitted.

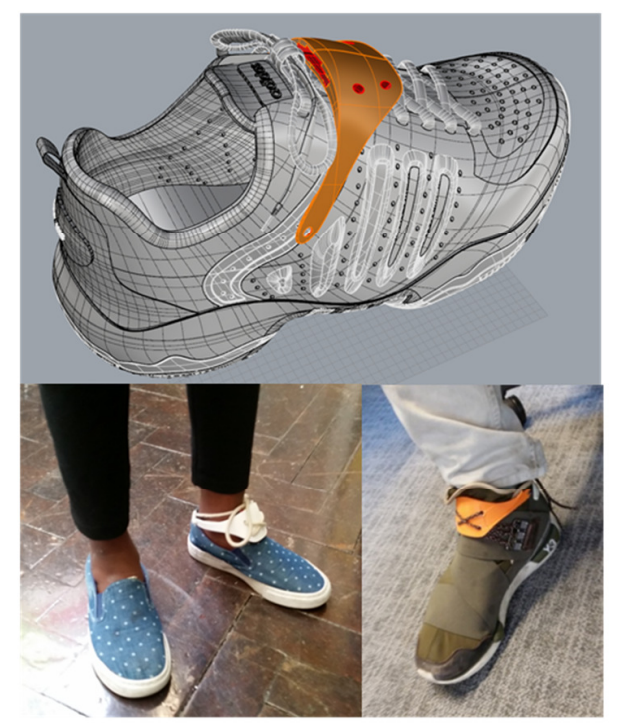

Figure 5: 3D printed show-flap protective shell for IMU

Group structures were identified based on betweenness centrality, using the Girvan-Newmann algorithm [4] implemented in NetworkX [5]. The class social network based on these nominations is shown in Figure 6. As Figure 6 shows, strong sex segregation is evident in the class network.

The sociometric network was also used to explore the social connectedness of the children by examining the number and strength of nominations each child received. We examined the number of nominations received (in-degree); in-degree weighted by preference ranking $\left(1^{\text {st }}=3,2^{\text {nd }}=2,3^{\text {rd }}=1\right)$; and reciprocated nominations (sum of edges between pairs of nodes).

\section{ANALYSES}

Once we had created the bespoke sensors and successfully used them to collect data under the piloting, baseline, and intervention conditions, we addressed our second aim: to develop ways to use the sensor data to characterise social play behaviours. In this section, we report two ways in which we approached this aim. Firstly, we discuss the development of spatio-temporal metrics matched to social gameplay type and, secondly, we discuss methods for deriving an affiliation network using measures of movement similarity between players.

\subsection{Types of Social Play}

Based on the video recordings and field observations, we observed and noted three main social play types that our method would need to characterise: (i) Hangout Play, (ii) Pursuit Play and (iii) Focal Play.

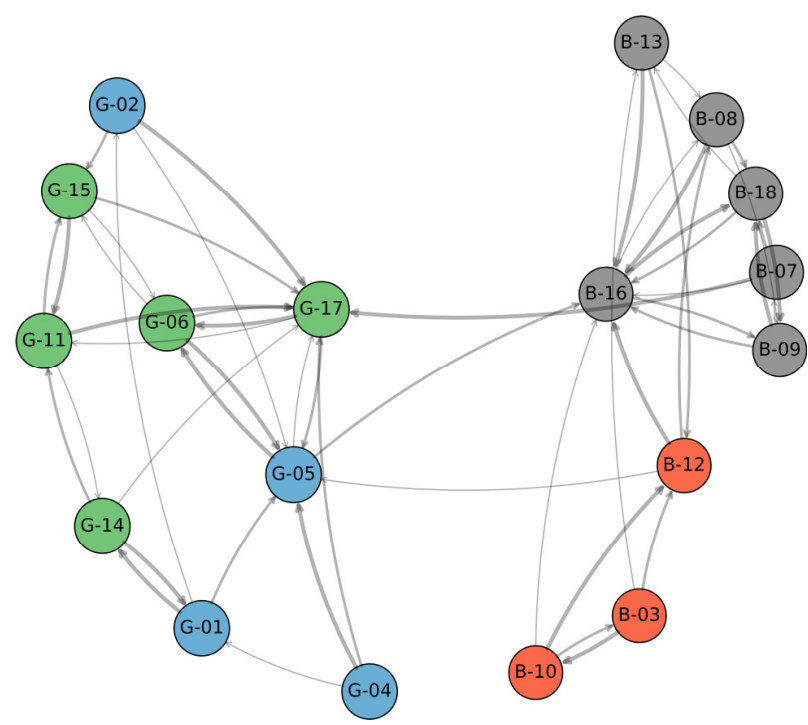

Figure 6: Sociogram of the class network. Nodes labelled Gand B- represent girls and boys respectively. Nodes with similar colours are communities detected after the 2 nd iteration of the Girvan-Newmann algorithm. The 1st iteration detected the gender segregation that is clearly seen from the structure of the graph.

\subsubsection{Hangout Play}

In hangout play, two or more children spend time together close to each other. They may stay in same location or move to different locations, so this form of play is primarily characterised by the duration of collocation, not where that collocation occurs. Hangout play is identified using a metric that involves the warping of point-to-point distances between pairs of individuals over a sliding window of time. Figure 7 shows two synthetic traces that represent an example of hangout play, coupled with the point-to-point distances between the individuals.

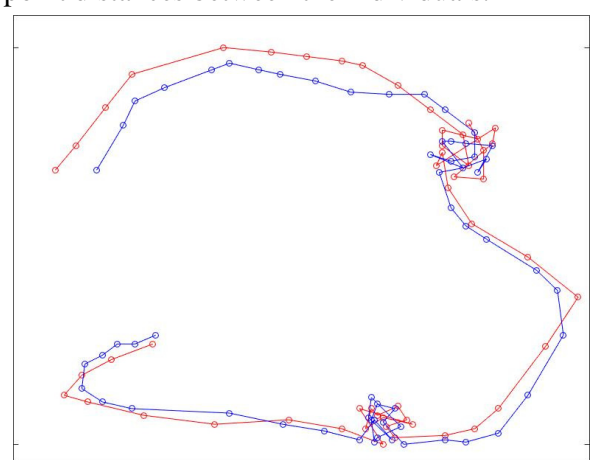

Figure 7: Hangout Play Example 


\subsubsection{Pursuit Play}

In pursuit play, two or more children follow each other in a chase; whilst they follow similar paths, they need never be collocated. In other words, there is spatial similarity with variable temporal lag. Figure 8 shows a synthetic example of pursuit play.

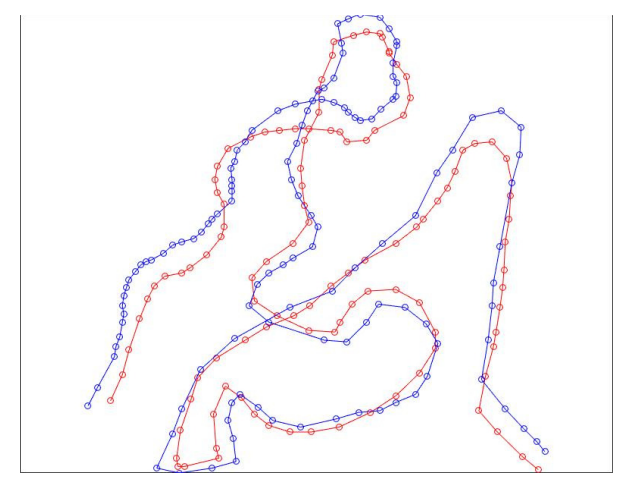

Figure 8: Pursuit Play Example

\subsubsection{Focal Play}

In focal play, two or more children visit the same location at different times. Examples include children bringing materials from different places to a shared location in pursuit of some common goal such as creating a structure. Other examples include ball games like basketball or football in which children follow the ball and return to the goal area at different times. Due to their unsynchronised returns to the focal point, point-to-point distances do not represent the associations in this game type (shown in Figure 9). Similarly, curve-matching based on asynchronous warping of area or distance is not representative of the associations in focal play.

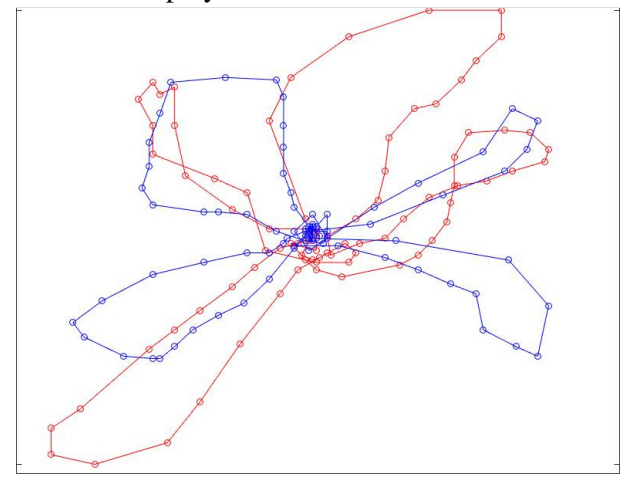

Figure 9: Focal Play Example

\subsection{Curve Similarity Matching for Gameplay Movement}

In this section, we propose and formulise different spatiotemporal metrics to measure the associations between children engaged in each type of play. The metrics in this section are chosen to reflect and cover the three play types defined and described in Section 3.1. The play types were defined based on our review of video files and notes taken by the play workers.

\subsubsection{Definitions}

Here, curves $\mathrm{A}$ and $\mathrm{B}$ are considered polygonal chains where $A=[0, m] \rightarrow \mathbb{R}^{2}$ and $|A|=m$ and $B=[0, n] \rightarrow \mathbb{R}^{2}$ and for $i=\{0, \ldots, n-1\}$ and $|B|=n . A_{i}$ (and $B_{i}$ ) denotes the segment between ith and (i+1)th points. $\psi_{A_{i}}$ denotes the azimuth angle of segment $A_{i} .\left(x_{i}, y_{i}\right)$ are polygon points of $A_{i}$ in local Cartesian coordinates converted from longitude and latitude points from GNSS results.

\subsubsection{Warping Turning Distance}

The curve trajectories of children in the playground are deducted as polygon chains synchronised by GNSS accurate real time. Conventional curve matching methods e.g. Arkin et al [8] using Warping Turning Distance (WTD) defined as

$$
W T D(A, B)=\min _{\substack{\theta \in \mathbb{R} \\ u \in[0,1]}}\left[\int_{0}^{1}\left(\psi_{A}(s)-\psi_{B}(s+u)+\theta\right)^{2} d s\right]^{\frac{1}{2}}(1)
$$

where $\theta$ is the optimal azimuth angle (orientation) and $u$ is the optimal starting point for best match between the two polygons. While such methods are useful for shape similarity and classification they may not represent the gameplay types we need to quantify in this work.

\subsubsection{Warping Euclidean Distance}

Similarly, we considered Warping Euclidean Distance (WED) [8], which can be defined for spatial curve matching defined as

$$
\begin{gathered}
W E D(A, B)=\min _{u \in[0,1]}\left[\int_{0}^{1}\left(x_{1}(s)-x_{2}(s+u)\right)^{2}+\right. \\
\left.\left(y_{1}(s)-y_{2}(s+u)\right)^{2} d s\right]^{\frac{1}{2}}
\end{gathered}
$$

However, this method is not useful in the case of pursuit play. In pursuit play, two children follow each other with a time delay and may not be collocated at any time. The time lag between them can adversely influence the WED metric.

\subsubsection{Warping Fréchet Distance}

We considered using the Fréchet distance as a basis for adding links in the play network. In Fréchet distance, the pair of contributing points sweeps continuously on each curve considering the flow and the ordering of the curve points [6, 7]. Since the rate of travel for either point may be non-uniform, Warping Fréchet Distance (WFD) is a more suitable method for curve similarity in ordered (synchronised) polygon chains in our case study. The Fréchet metric is defined as infimum of

$$
W F D(A, B)=\inf _{\alpha, \beta} \max _{u \in[0,1]}[\mathcal{D}(A(\alpha(u)), B(\beta(u))]
$$

where $\alpha(\mathrm{u})$ and $\beta(\mathrm{u})$ are continuous and increasing functions with $\alpha(0)=0, \alpha(1)=m, \beta(0)=0$ and $\beta(1)=n$ only. $\mathcal{D}$ is the Euclidean distance between the sweeping points. In practice WFD can be computed by dynamic programming algorithms [16].

Figure 10 shows examples of WED and WFD for Pursuit Play showing the efficiency of WFD over WED in gameplay similarity measurement. 
Table 1 shows the potential usefulness of the methods described above for gameplay type defined in section 3 . The comparison is based on ground truth data shown in Figures 7, 8 and 9. Since the distances are computed and compared for ground truth data, smaller numbers show better representation of the game play association.

Table 1: Comparison of Distance Metrics vs Gameplay Types

\begin{tabular}{ccccc}
\hline \multirow{2}{*}{$\begin{array}{c}\text { Distance } \\
\text { Metric }\end{array}$} & Hangout & Pursuit & Focal & Random \\
\hline WTD (rad) & 2.461 & 1.543 & 3.981 & 14.322 \\
WED (m) & 1.086 & 2.699 & 5.001 & 13.511 \\
WFD (m) & 0.629 & 1.239 & 1.140 & 2.246 \\
\hline
\end{tabular}
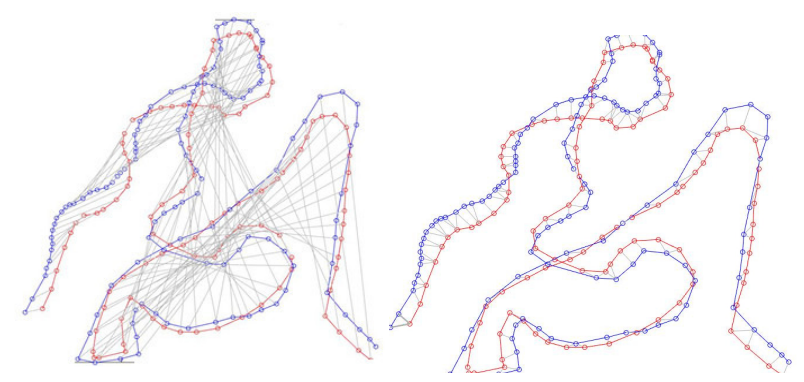

Figure 10: Warping Euclidean Distance (left) compared to Fréchet Distance Metric (right) in Pursuit Play

\subsection{Creating a Movement-based Social Affiliation Network}

Considering the WFD method as likely to be the most informative irrespective of play type, the second piece of analysis involved creating a social affiliation network summarising spatio-temporal data from each child across the 10 baseline and 10 intervention episodes, and using similarity between pairs of children to add links between nodes as described in 3.3.1.

\subsubsection{Gameplay Similarity Decision Problem}

Let $\mathbb{G}$ be the set of continuous sliding windows $S_{j}^{t}$ of t seconds of movement data subsets (polygon chains) over the course of playground playtime sessions:

$$
\mathbb{G}=\left\{S_{j}^{t} \mid j=1 . . p, A_{j}=f\left(S_{j}^{t}\right)\right\}
$$

For each two children and on each $S_{j}^{t}$ we define the following decision problem:

Given two gameplays and a threshold $\varepsilon \geq 0$ decide whether

$$
F_{\varepsilon}=\left\{(s, t) \in[0, m] \times[0, n] \mid W F D\left(A^{s}, B^{t}\right) \leq \varepsilon\right\}
$$

$F_{\varepsilon}$ describes all pairs of sliding windows $S_{j}^{t}$ and their corresponding polygon chains $A_{j}$ whose distance is at most $\varepsilon$. For $r$ children in the class we calculate the Overall Affiliation Matrix (OAM) which is a symmetric $r \times r$ matrix $\mathcal{W}$ where the weights $\mathcal{W}_{i, j}$ are

$$
\mathcal{W}_{i, j}=\left|F_{\varepsilon}^{\mathrm{i}, \mathrm{j}}\right|
$$

\section{RESULTS}

The OAM was used to calculate the affiliation networks graph based on averages over the baseline and intervention episodes. We also represent these data as dendrograms and clustergrams as this allows for visualisation of hierarchical clusters (sub-group formations) in the class group. We present these results descriptively before discussion of their possible interpretation with respect to our research aims in section 5 .

Figures 11 and 12 show the class networks for baseline and intervention.
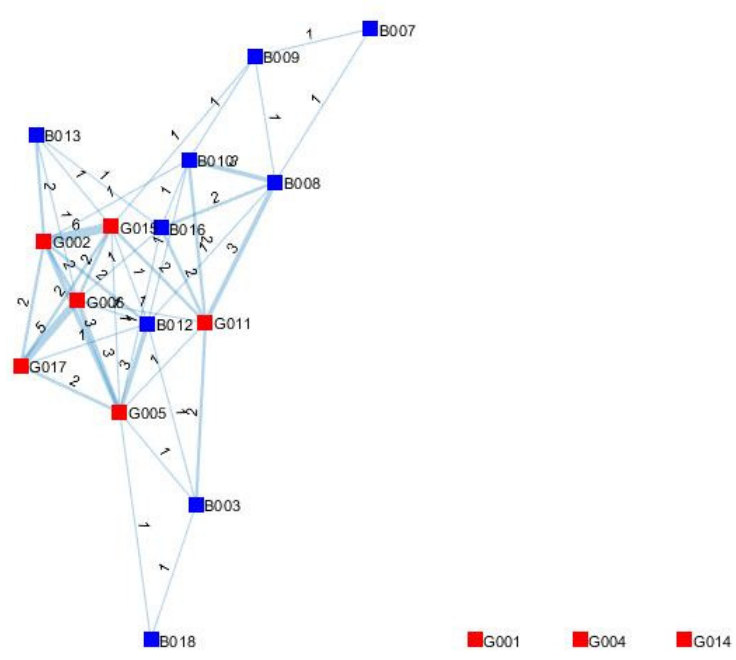

Figure 11: Network Graph Baseline (blue squares are boys and red squares are girls)

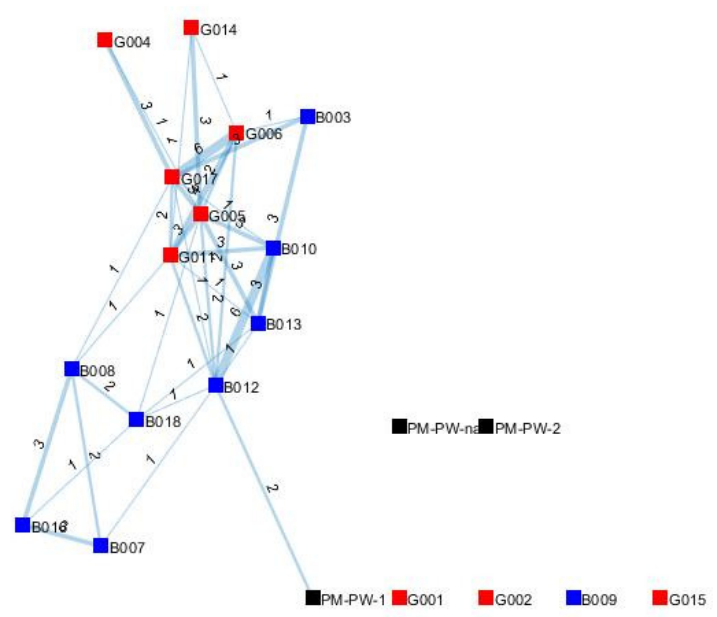

Figure 12: Network Graph Intervention (blue squares are boys, red squares are girls and black are playworkers)

These network graphs show the overall affiliation matrices at the least permissive threshold for establishing a link. A 'spring' layout is used for the visualisation, meaning nodes placed closer together have stronger connections. Nodes appear isolated from 
the main graph when they were not sufficiently similar to any other child to add a link to the OAM as described in 3.3.1 above. To clarify the information contained in the networks, we also present the information as clustergrams and dendrograms. Figure 13 and 14 show the class clustergrams for baseline and intervention data. The playworker data have also been included for the intervention matrix.

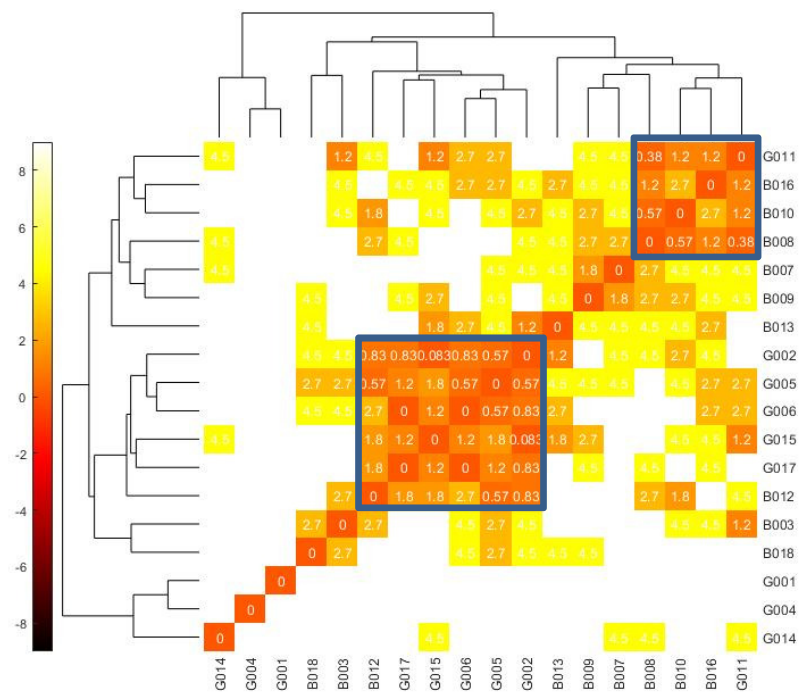

Figure 13: Clustergram and dendrogram of baseline data. B=Boy, $\mathbf{G}=$ Girl

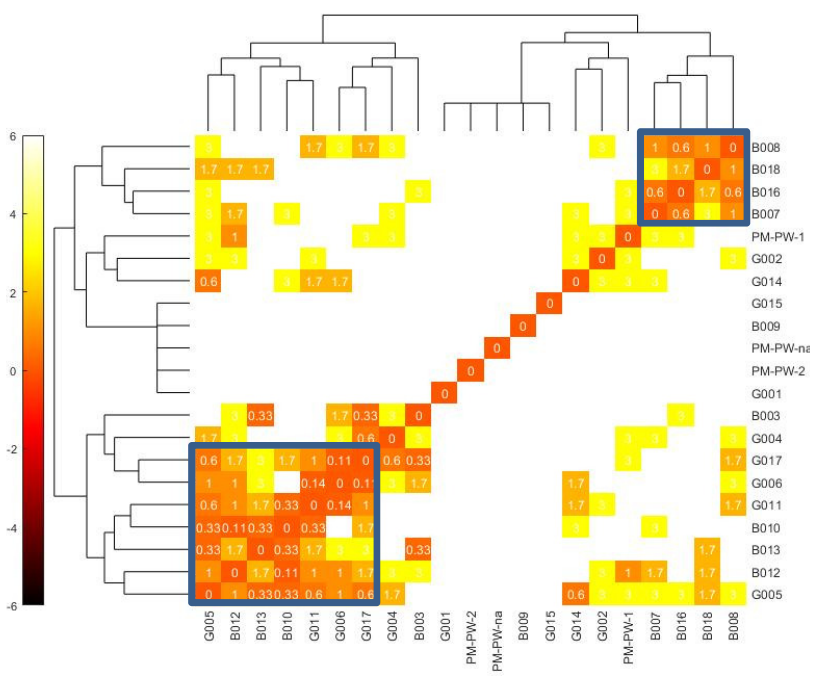

Figure 14: Clustergram and dendrogram of intervention data. B=Boy, G=Girl, PM-PW=Playworker

The hierarchical structures of the group are shown in the dendrogram at the top of the diagram (repeated also on LH side). The coloured squares represent the strength of association in the OAM between each pair of participants as described in 3.3.1
(White/yellow are the 'weakest' connections, darker orange the 'stronger' ones).

\section{ANALYSIS AND DISCUSSION}

In this case study we have demonstrated the development and successful implementation of a bespoke sensor for tracking children's movements during episodes of unstructured social play. Importantly, the method appears to be ethically acceptable to children and families. Indeed, discussions with the participating school showed us that they were more comfortable with the GNSS/IMU methods of data capture than with video, as child data could more readily be anonymised.

The method we developed did not prove inhibitory to social play behaviours and children quickly habituated to the wearables. Children enjoyed participating in the assembly and decoration of the wearable caps and this turned out to be a good strategy for engaging the children in the research. The case study therefore illustrates that we met our objective to construct a user-friendly system of wearable sensors to track children's movements and interactions on the playground.

Our second objective was to develop ways to use the resulting sensor data to characterise social play behaviours. This is an important consideration when deciding whether or not it would be worth developing the method beyond this initial case study. The identification of three social movement patterns of interest in this paper (section 3.1) provides a useful reference point for future research.

The Overall Affiliation Matrices (visualised as clustergrams and networks in section 4), show there is interesting social information contained in the trajectories of children in a playspace. For example, in the baseline condition network there is evidence of sex segregation, something we also observed in the nomination network (see section 2.4). At the first level we observe one main cluster composed mainly of boys with one girl included, and a second (larger) cluster composed mainly of girls with one boy included. A third set of weakly connected girls is also observed. It is interesting to note that node G-004 is an isolated node in the baseline network (Fig. 13); this child is also isolated in the sociometric network (i.e. she received no incoming nominations in the directed graph, see Fig. 6).

In the intervention network it is encouraging to see that the playworkers are represented by isolated or very weakly connected nodes, as their role was intended to be as 'hands-off' as possible from the children's play. As in the baseline condition (Fig. 13), G001 is poorly connected, as are children B009, G015 and G002. Interestingly, in the upper right-hand corner of the clustergram, we see a more sharply defined single-sex cluster of boys in the intervention data. Matching this to our fieldnotes we see that this corresponds to what we have named 'the football effect.' When the play materials were available to the children, a small group of boys chose to engage in this activity in preference to all others. The second cluster at the first level of the intervention data is also of interest in that here we see more integration between the sexes, four boys clustered alongside five girls. In this condition we see the previously isolated child G004 is included in the cluster. 
Informal observations based on fieldnotes suggest that this increased integration occurs during collaborative interactions with the loose parts materials.

From this exploratory case study, it is clear that using curve similarity to define links in an affiliation network is a potentially useful tool for exploring social structures and dynamics as they occur during naturalistic play. This could help to test hypotheses in developmental social psychology that have previously been impractical to explore, especially those that apply to whole group behaviour. For example, the hypothesised influence of play type and activity levels on the emergence of sex segregated behaviour in pre-pubescent groups could be studied [14], or theories of distinct social-behavioural phenotypes associated with different neurodevelopmental conditions could be tested [15]. The method also has potential as an objective test of change in social behaviour following intervention.

\section{CONCLUSIONS}

In this work, we created and successfully implemented a new method of studying children's social group behaviour at playtime using movement and positioning data. Bespoke wearable sensors for motion capture, high-accuracy localisation and game play analysis have been designed and developed. Empirical data using a class of 18 children were collected in 27 sessions. Three main types of playground game play were identified using video data and fieldnotes. We have proposed and compared different observation metrics to define similarity between children during these different types of playground social play. Sociometric analyses were also conducted and used for comparison and validation.

Experimental results were demonstrated using affiliation network graphs and clustergrams. Sex segregation in both sociometric and in sensor derived network results were observed. This gives a certain face-validity to the methodology as sex segregation in play behaviour is a well-documented phenomenon [6]. However, both 'the football effect' and the more integrated group seen in the intervention condition, indicate possible environmental effects on sex segregation during play. It is important to reiterate that, at this stage in the development of our methodology, we did not aim formally to test specific hypotheses. Rather we aimed to explore whether we could conclude that our method is suitable for capturing differences in social behaviour as it occurs during different play environments. The results from this case study suggest the method does have promise in this direction. The novel contribution of this work lies in the development of an objective and ethically acceptable methodology for using spatial and temporal information to further understanding of peer relationships in free-play settings.

The next steps include (i) examining associations between traditional measures of social ability or social status and those derived from the properties of the OAM interaction network; (ii) investigating activity levels \& group formation processes; (iii) developing machine learning classifiers for different play types, validated against video; and iv) investigating whether observed relations hold in different play environments such as urban or rural settings.

\section{ACKNOWLEDGMENTS}

This research was funded by the UK Economic and Social Research Council (ESRC) Transformative grant scheme: ES/N006577/1. Additional funding from LEGO Foundation supported dissemination. Thanks to Learning through Landscapes, Children's Scrapstore, Community Playthings, Play England and Touchwood for donations of materials, time, and excellent advice about play. Thanks to Richard Barry for coordinating the intervention. Thanks to Rachel England, Anna Vidos, Sam Hailes, and other student volunteers for valuable help with data collection. Thanks to Belle for BSL interpretation. Thanks to the children and teachers at the participating primary school.

\section{REFERENCES}

[1] Baines, E. and Blatchford, P. 2009. Sex differences in the structure and stability of children's playground social networks and their overlap with friendship relations. British Journal of Developmental Psychology. 27, 743760. DOI:https://doi.org/10.1348/026151008X371114.

[2] Coie, J.D. et al. 1982. Dimensions and types of social status: A cross-age perspective. Developmental Psychology. 18, 4 (1982), 557-570. DOI:https://doi.org/10.1037//0012-1649.18.4.557.

[3] Gibson, J.L. et al. 2017. A Systematic Review of Research into the Impact of Loose Parts Play on Children's Cognitive, Social and Emotional Development. School Mental Health, 9, Issue 4, 295-309.

[4] Girvan, M. and Newman, M.E.J. 2002. Community structure in social and biological networks. PNAS.

[5] Hagberg, A. a. et al. 2008. Exploring network structure, dynamics, and function using NetworkX. Proceedings of the 7 th Python in Science Conference (SciPy2008) (2008).

[6] Martin, C.L. and Fabes, R.A. 2001. The stability and consequences of young children's same-sex peer interactions. Developmental psychology. DOI:https://doi.org/10.1037/0012-1649.37.3.431.

[7] Moreno, A. and Poppe, R. 2013. "You're it!": Role Identification using Pairwise Interactions in Tag Games. IEEE Conference on Computer Vision and Pattern Recognition, 657-662. DOI:https://doi.org/10.1109/CVPRW.2013.99.

[8] Pellegrini, A.D. and Bohn, C. 2005. The role of recess in children's cognitive performance and school adjustment. Educational Researcher. 34, 1 (2005), $13-19$.

[9] Veiga, G. et al. 2015. Social Competence at the Playground: Preschoolers During Recess. Infant and Child Development.

[10] Aharon Weissbrod, et al. 2013. Automated long-term tracking and social behavioural phenotyping of animal colonies within a semi-natural environment. Nature communications. 4, May.

[11] Woolford, K. 2014. Capturing Human Movement in the Wild. Proceedings of the 2014 International Workshop on Movement and Computing, New York, NY, USA, 19:19--19:24

[12] S. Ayub, B. M. Heravi, A. Bahraminasab, B. Honary. 2012. Pedestrian direction of movement determination using smartphone', Next Generation Mobile Applications, Services and Technologies (NGMAST), 2012 6th International Conference on, IEEE, 64-69.

[13] M. Gupta, C. Holloway, B. M. Heravi, S. Hailes. 2015. A comparison between smartphone sensors and bespoke sensor devices for wheelchair accessibility studies, Intelligent Sensors, Sensor Networks and Information Processing (ISSNIP), 2015 IEEE Tenth International Conference on, 1-6.

[14] Pellegrini, A. D., Long, J. D., Roseth, C. J., Bohn, C. M., \& Van Ryzin, M. 2007. A short-term longitudinal study of preschoolers'(Homo sapiens) sex segregation: The role of physical activity, sex, and time. Journal of Comparative Psychology, 121(3), 282.

[15] J. Gibson, J. Hussain, S. Holsgrove, C. Adams, J. Green. 2011. Quantifying peer interactions for research and clinical use: The Manchester Inventory for Playground Observation. Research in Developmental Disabilities, 32(6), 24582466.

[16] H. Alt, A. Efrat, G. Rote, C. Wenk. 2003. "Matching planar maps", Journal of algorithms, 262-283. 